\title{
Meta-Analysis of Risk Factors of Vascular Cognitive Disorder in Acute Cerebral Infarction Patients
}

\author{
Yan Liang, Xin Ao* \\ Department of Medicine, Yangtze University, Jingzhou, China \\ Email: *cjdxhlx@126.com
}

How to cite this paper: Liang, Y. and Ao, X. (2020) Meta-Analysis of Risk Factors of Vascular Cognitive Disorder in Acute Cerebral Infarction Patients. Yangtze Medicine, 4, 97-113.

https://doi.org/10.4236/ym.2020.42010

Received: May 15, 2019

Accepted: June 26, 2020

Published: June 29, 2020

Copyright $\odot 2020$ by author(s) and Scientific Research Publishing Inc. This work is licensed under the Creative Commons Attribution International License (CC BY 4.0).

http://creativecommons.org/licenses/by/4.0/

\begin{abstract}
Objectives: To identify the main risk factors of vascular cognitive impairment in patients with acute cerebral infarction by Meta-analysis, and provide references for the effective prevention of the cognitive impairment in stroke patients. Methods: To retrieve the observational research literatures that refer to the risk factors of vascular cognitive impairment in patients with ischemic stroke, which are published on China National Knowledge Infrastructure (CNKI), Wanfang and Weipu Chinese databases. The screening and data extraction of these literatures are independently completed by two researchers, who also give the quality evaluation of the literatures according to the evaluation criterion of the Australian JBI Evidence-Based Health Care Center. Then, Meta-analysis is conducted by using Revman5.3 software. Results: There are twenty-eight articles selected from 1507 literatures, with a total of 10,711 cases and 50 risk factors included. Among them, there are combined effects of ten factors which have statistical significance, such as infarction area, alcohol consumption, smoking, hyper homocysteinemia, hypertension, diabetes mellitus, age, history of cerebral infarction, hyperlipoidemia and education level. The relational merging OR value and 95\% CI between the type-variable factors and cognitive impairment are 3.25 (1.84, 5.76); 2.98 (2.58, 3.45); 2.79 (1.69, 4.59); 2.35 (1.93, 2.85); 2.25 (1.86, 2.71); 2.14 (2.10, 2.18); 1.82 (1.62, 2.03); $1.54(1.24,1.92) ; 1.45$ (1.34, 1.56); 0.83 (0.78, 0.89). Conclusion: Infarction area, alcohol consumption, smoking, hyper homocysteinemia, hypertension, diabetesmellitus, age, history of cerebral infarction, hyperlipoidemia and low education level are the main risk factors for vascular cognitive impairment in patients with acute cerebral infarction. Clinical nursing staff should include it into the routine assessment of patients with acute cerebral infarction and actively prevent and intervene.
\end{abstract}




\section{Keywords}

Cerebral Ischemia, Vascular Cognitive Impairment, Risk Factors, Meta-Analysis

\section{Introduction}

Vascular Cognitive Impairment (VCI) is a major clinical syndrome with varying degrees of cognitive dysfunction [1]. These symptoms are caused by risk factors of cerebrovascular diseases, various obvious or not obvious cerebrovascular diseases. Among them, acute cerebral infarction (also known as acute ischemic stroke) is one of the most common causes of VCI. At present, the acute cerebral infarction has been the second leading cause of the death people over 60 years old in the whole world [2], also with a high incidence rate in China [3]. Once cognitive dysfunction occurs after cerebral infarction [4], the patient's life will be impaired significantly, which will bring a great burden to families and society. Therefore, early detection and elimination of risk factors for VCI in patients with acute cerebral infarction are very important for the effective prevention and treatment of VCI. However, although there are many studies on the risk factors of VCI in cerebral infarction, the results are still inconsistent. There are also some limitations to explore the risk factors, lacking of comprehensive systematic research. In the view of the above reasons, the study intends to summarize the vital risk factors of VCI in patients with acute cerebral infarction, using the meta-analysis to analyze the data from those high-quality research literatures extracted and integrated from the documents published, so as to provide scientific guidance for the clinical nursing and prevention.

\section{Materials and Methods}

\subsection{Strategies of Search}

By means of combining the subject words and keywords, this study has retrieved the literatures (from the China National Knowledge Infrastructure (CNKI), Wanfang Database, and Weipu Database) about case-control studies, cohort studies and cross-setional studies on risk factors of VCI in those occurring acute cerebral infarction. Those unpublished or gray literatures have been excluded from this study. The keywords are the combination of subject words and free words, and the Chinese retrieval strategy is as follows: ischemic stroke, vascular cognitive impairment, risk factors, influencing factors, case-control studies, cohort studies and epidemiological studies. In addition, the references included in the literature were searched by hand.

\subsection{Inclusive and Exclusive Criteria}

The inclusive criteria for the literature are as follows. Firstly, the study design involves the literatures about cohort studies, analytical cross-sectional studies 
and epidemiological studies. Secondly, the objects of study are those patients over 18 years old with cerebral infarction diagnosed by the clinical manifestation combined with imaging CI or MRI (Magnetic Resonance Imaging). Thirdly, the literatures with OR (Odds ration) value, RR (Relative risk) value and 95\% CI (confidence interval) can be provided. Fouthly, Chinese publication, the starting and ending time of all retrieval documents is from the construction of the database to August 2018.

The exclusive criteria for the literature are as follows. Firstly, the objects of study do not include those who suffer from Alzheimer's disease, cerebral hemorrhage and vascular dementia. Secondly, those literatures without clear diagnostic tools and methods for cognitive dysfunction are excluded. Thirdly, the literatures with poor quality, incomplete date and indefinite diagnosis are excluded.

\subsection{Extraction of Literatures}

Two researchers independently completed the screening of the literatures and the extraction of the data. When there were disagreements, two researchers discussed together to find the solution. If necessary, the third researcher was consulted to make a decision on the results of the inclusion or exclusion. The flow chart of literature screening is as follows:

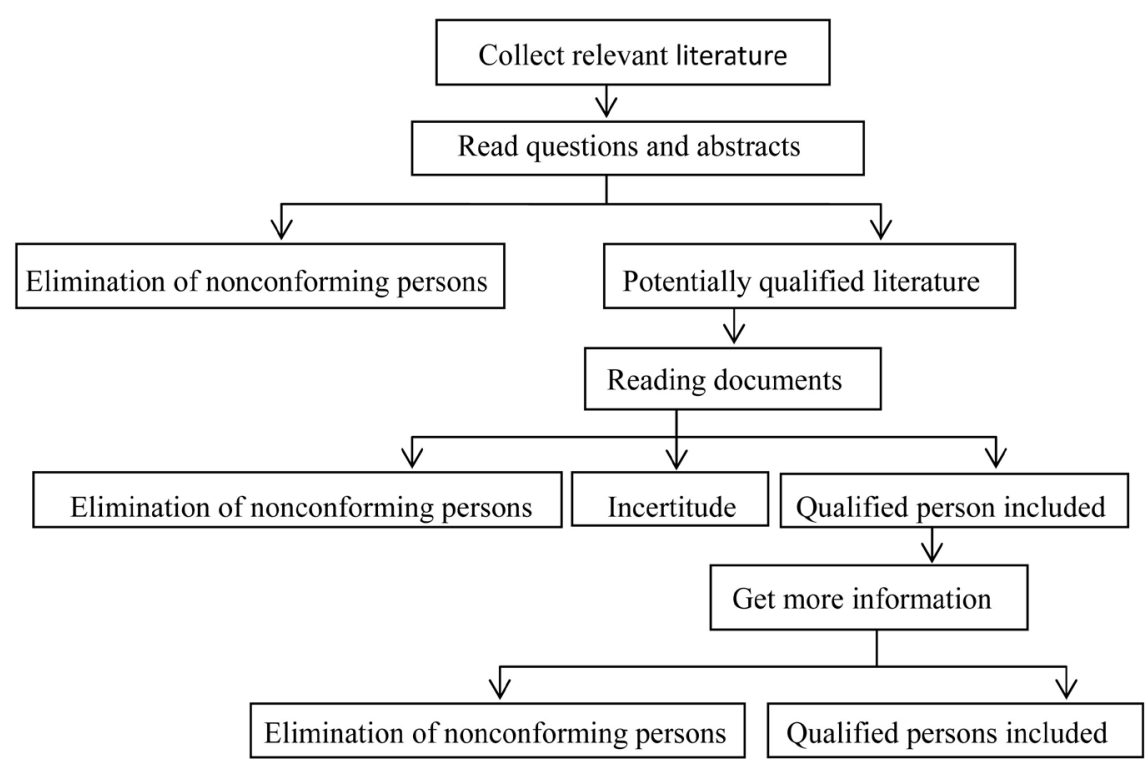

\subsection{Quality Evaluation of Literatures}

Case-control studies, cohort studies, and analytical cross-sectional studies are evaluated according to the standards of the Australian JBI Evidence-Based Health Care Center (2016 version). There are 10 items included in case-control studies, 11 items in cohort studies and 8 items in analytical cross-sections, with a judgment of "yes", "no", "unclear", and "not applicable" made for each item. (Evaluation criteria are shown in Tables 1-3.) 
Table 1. Authenticity evaluation of case-control study in JBI evidence-based Health Care Center, Australia.

\begin{tabular}{l} 
Evaluation Item \\
\cline { 2 - 3 } Yes No Unclear Not applicable \\
1) Are the factors in the case group and the control \\
group comparable except for suffering from \\
different diseases? \\
2) Is the match between the case group and the \\
control group appropriate? \\
3) Are the same criteria used to recruit case group \\
and control group? \\
4) Is the exposure factor measured by a standard, \\
effective, and credible method? \\
5) Is the same method used to measure the exposure \\
factors of the case group and the control group? \\
6) Have you considered confounding factors? \\
7) Have you taken measures to control the \\
confounding factors? \\
8) Is the standard, effective, and credible \\
method used to measure outcomes? \\
9) Is the exposure time long enough? \\
10) Is the data analysis method appropriate?
\end{tabular}

Table 2. Authenticity evaluation of cohort study in JBI Evidence-based Health Care Center, Australia.

\begin{tabular}{lllll}
\hline \multirow{2}{*}{ Evaluation Item } & \multicolumn{3}{c}{ Evaluation Results } \\
\cline { 2 - 3 } & Yes & No & Unclear & Not applicable \\
\hline
\end{tabular}

1) Does the study group have similar characteristics and are derived from the same research population?

2) Are the exposure factors measured in the same way and assigned to the exposed and non-exposed groups?

3) Is the evaluation method for exposure factors effective and credible?

4) Have you considered confounding factors?

5) Have you taken measures to control the confounding factors?

6) Does it describe that the study subjects do not show an observation outcome at the beginning of the exposure or study?

7) Is the evaluation method of the outcome indicator effective and credible?

8) Do you report the follow-up time and was the follow-up time long enough to observe the appearance of the outcome? 


\section{Continued}

9) Is the follow-up complete, if not, whether to describe and analyze the cause of the lost visit?

10) Have you taken measures to deal with the loss of follow-up?

11) Is the data analysis method appropriate?

Table 3. Authenticity evaluation of analytical cross-sectional study in JBI Evidence-based Health Care Center, Australia.

\begin{tabular}{|c|c|c|c|c|}
\hline \multirow{2}{*}{ Evaluation Item } & \multicolumn{4}{|c|}{ Evaluation Results } \\
\hline & Yes & No & Unclear & Not applicable \\
\hline $\begin{array}{l}\text { 1) Is the inclusion criteria for the sample } \\
\text { clearly defined? }\end{array}$ & & & & \\
\hline $\begin{array}{l}\text { 2) Has the research object and research site } \\
\text { been described in detail? }\end{array}$ & & & & \\
\hline $\begin{array}{l}\text { 3) Does the measurement method of exposure } \\
\text { factors have reliability and validity? }\end{array}$ & & & & \\
\hline $\begin{array}{l}\text { 4) Is there an objective and consistent standard } \\
\text { for the definition of disease or health problems? }\end{array}$ & & & & \\
\hline 5) Have you identified a confounding factor? & & & & \\
\hline $\begin{array}{l}\text { 6) Are measures taken to control confounding } \\
\text { factors? }\end{array}$ & & & & \\
\hline $\begin{array}{l}\text { 7) Does the measurement method of the } \\
\text { outcome indicator have reliability and validity? }\end{array}$ & & & & \\
\hline 8) Is the data analysis method appropriate? & & & & \\
\hline
\end{tabular}

\subsection{Statistical Analysis Methods}

Comprehensive analysis of the research data was performed with RevMan5.3 software. The heterogeneity test was carried out first. It is considered that there is homogeneity between the studies (in the case of $P>0.10$ and $\mathrm{I}^{2}<50 \%$ ), which is described as the fixed effect model. However, there is heterogeneity between the studies (in the case of $P \leq 0.10$ and $\mathrm{I}^{2}>50 \%$ ), which needs to look for the causes of heterogeneity and exclude the literatures with unreasonable design. If it is still heterogeneous, describe it as a random effect model. Then, the effect quantity in each document is weighted and combined, the combined OR value and $95 \% \mathrm{CI}$ of the literatures are calculated and output with OR as the effect quantity index. According to the results of forest map analysis, in the case of $\mathrm{OR}>1$, the exposure factor is the risk factor of the disease, in the case of $\mathrm{OR}<1$, it indicates that the exposure factor is negatively correlated with the disease, because the exposure factor is the protective factor of the disease.

\section{Results}

\subsection{Results of Literature Search}

A total of 1507 literatures were retrieved through the literature database, from 
which, 1281 articles were excluded on account of not meeting the inclusive and exclusive criteria according to their topics and abstracts, 226 articles met the inclusion criteria. After reading the full text further, 198 articles were excluded and 27 articles were selected finally.

\subsection{Characteristics of the Selected Literatures}

Among the 27 articles selected, 1 about cohort study, 8 about analytical cross-sectional study and 18 about case-control studies. There are totally 10,711 samples in this study, including 6639 cases for the normal cognitive sample group and 4072 cases for the cognitive impairment group.

\subsection{Quality of the Selected Literatures}

According to the evaluation criteria of The Australian JBI Evidence-Based Health Care Center, there is 1 article about cohort study, 8 articles about analytical cross-sectional study with A-levels, and 18 articles about case-control study with 12 of A-levels and 6 of B-levels.

The evidence recommendation level table is shown in Table 4.

The basic conditions and quality evaluation results of the literature are shown in Tables 5-7.

Table 4. JBI evidence recommendation level (version 2010).

\begin{tabular}{cr}
\hline Recommended level & Rationality/suitability/clinical significance/effectiveness \\
\hline A level recommendation & The evidence is strong and can be applied. \\
B level recommendation & Evidence moderate support, consider application \\
C level recommendation & The evidence does not support it. \\
\hline
\end{tabular}

Table 5. Basic information and quality evaluation results of the cohort study literature included in the study.

\begin{tabular}{ccccccccccccccccccc}
\hline Inclusive study & $\begin{array}{c}\text { Abnormal } \\
\text { group (case) }\end{array}$ & $\begin{array}{c}\text { Normal } \\
\text { group (case) }\end{array}$ & (1) & (2) & (3) & (4) & (5) & (6) & (7) & (8) & (9) & (10) & (11) & Grade \\
\hline $\begin{array}{c}\text { Dong Xinzhe } \\
{[5] 2017}\end{array}$ & 47 & 69 & Yes & Yes & Yes & Yes & Yes & Yes & Yes & Yes & Yes & Yes & Yes & A & & &
\end{tabular}

Table 6. Basic information and quality evaluation results of cross-sectional study included in the study.

\begin{tabular}{cccccccccccc}
\hline Inclusive study & abnormal group (case) & normal group (case) & (1) & (2) & (3) & (4) & (5) & (6) & (7) & (8) & Grade \\
\hline Feng Qingchun [6] 2017 & 210 & 225 & Yes & Yes & Yes & Yes & Yes & Yes & Yes & Yes & A \\
Zhang Xiaowei [7] 2014 & 93 & 66 & Yes & Yes & Yes & Yes & Yes & Yes & Yes & Yes & A \\
Wang Yan et al. [8] 2013 & 220 & 94 & Yes & Yes & Yes & Yes & Yes & Yes & Yes & Yes & A \\
Wang Jin et al. [9] 2017 & 90 & 451 & Yes & Yes & Yes & Yes & Yes & Yes & Yes & Yes & A \\
Zhou Xiaosheng [10] 2014 & 118 & 316 & Yes & Yes & Yes & Yes & Yes & Yes & Yes & Yes & A \\
Tu Qiuyun [11] 2011 & 288 & 401 & Yes & Yes & Yes & Yes & Yes & Yes & Yes & Yes & A \\
Liu Jing [12] 2015 & 1504 & 1496 & Yes & Yes & Yes & Yes & Yes & Yes & Yes & Yes & $A$ \\
Tian Qianqian [13] 2014 & 211 & 308 & Yes & Yes & Yes & Yes & Yes & Yes & Yes & Yes & A \\
\hline
\end{tabular}


Table 7. Basic situation and quality evaluation results of case-control study literature included in the study.

\begin{tabular}{|c|c|c|c|c|c|c|c|c|c|c|c|c|}
\hline Inclusive study & $\begin{array}{l}\text { Abnormal } \\
\text { group (case) }\end{array}$ & $\begin{array}{c}\text { Normal } \\
\text { group (case) }\end{array}$ & (1) & (2) & (3) & (4) & (5) & (6) & (7) & (8) & Grade & Inclusive study \\
\hline Yin Qi [14] 2014 & 68 & 82 & Yes & Yes & Yes & Yes & Yes & Yes & Yes & Yes & Yes & A \\
\hline Guo Xiaofeng [15] 2017 & 70 & 80 & Yes & Yes & Yes & Yes & Yes & Unclear & Yes & Unclear & Yes & B \\
\hline Sun Jiangli [16] 2015 & 183 & 532 & Yes & Yes & Yes & Yes & Yes & Unclear & Yes & Unclear & Yes & B \\
\hline Yin Songli [17] 2014 & 54 & 66 & Yes & Yes & Yes & Yes & Unclear & Unclear & Yes & Yes & Yes & B \\
\hline Chen Xiangliang [18] 2016 & 42 & 26 & Yes & Yes & Yes & Yes & Yes & Yes & Yes & Yes & Yes & A \\
\hline He Dan [19] 2017 & 76 & 44 & Yes & Yes & Yes & Yes & Yes & Yes & Yes & Yes & Yes & A \\
\hline Fan Yachao [20] 2012 & 68 & 75 & Yes & Yes & Yes & Yes & Yes & Yes & Yes & Yes & Yes & A \\
\hline Ning Fangbo [21] 2015 & 82 & 126 & Yes & Yes & Yes & Yes & Yes & Yes & Yes & Yes & Yes & A \\
\hline Xu Daijun [22] 2014 & 47 & 68 & Yes & Yes & Yes & Yes & Yes & Yes & Yes & Yes & Yes & A \\
\hline Chu Xinjuan [23] 2017 & 70 & 30 & Yes & Yes & Yes & Yes & Unclear & Unclear & Yes & Yes & Yes & B \\
\hline Mu Junshan [24] 2015 & 70 & 30 & Yes & Yes & Yes & Yes & Unclear & Unclear & Yes & Yes & Yes & B \\
\hline Wang Yanchao [25] 2017 & 48 & 72 & Yes & Yes & Yes & Yes & Yes & Yes & Yes & Yes & Yes & A \\
\hline Wu Jixia [26] 2018 & 132 & 358 & Yes & Yes & Yes & Yes & Yes & Yes & Yes & Yes & Yes & A \\
\hline Liu Yanjun [27] 2010 & 82 & 58 & Yes & Yes & Yes & Yes & Yes & Yes & Yes & Yes & Yes & A \\
\hline Zhu Yanxia [28] 2013 & 51 & 73 & Yes & Yes & Yes & Yes & Yes & Yes & Yes & Yes & Yes & A \\
\hline Gao Yuan [29] 2015 & 77 & 1279 & Yes & Yes & Yes & Yes & Yes & Yes & Yes & Yes & Yes & A \\
\hline Wang Dali [30] 2013 & 84 & 41 & Yes & Yes & Yes & Yes & Yes & Yes & Yes & Yes & Yes & A \\
\hline Sun Yao [31] 2018 & 60 & 80 & Yes & Yes & Yes & Yes & Yes & Unclear & Yes & Yes & Yes & B \\
\hline
\end{tabular}

\subsection{Merged Results of the Risk Factor Effect Quantity}

There are 50 risk factors were included in this study, of which, 10 risk factors were reported in 4 or more articles, 1 risk factor was reported in 3 articles, 11 risk factors were reported in 2 articles, and 28 risk factors were only reported in a single one. For this study, it selected totally 13 risk factors which were reported in 3 or more articles and also included in the systematic evaluation, which cover infarct size, alcohol consumption, smoking, high homocysteine, hypertension, diabetes, advanced age, history of stroke, hyperlipemia, and low education level. Among which, 3 articles that reported the infarct size was related to VCI in patients, which were all analyzed with meta-analysis. The merged results are $\mathrm{OR}=$ $3.25,95 \%$ CI $(1.84,5.76), P<0.0001, \mathrm{I}^{2}=0 \%$ (Figure 1), which reflect that the infarct size is highly correlated with VCI and is a risk factor for VCI in patients; 10 articles having reported the association of alcohol consumption and VCI, 7 are eligible for meta-analysis. The merged results show that drinking is a risk factor for VCI in patients with cerebral infarction (OR $=2.98,95 \%$ CI $(2.58$, $3.45), P<0.00001, \mathrm{I}^{2}=38 \%$ ) (Figure 2); 11 articles having reported the relationship of smoking and VCI, 4 articles are available for meta-analysis. The merged results are $\mathrm{OR}=2.79 .95 \% \mathrm{CI}\left(1.69,4.59, P<0.0001, \mathrm{I}^{2}=48 \%\right)$ (Figure 3), which show that smoking is also a risk factor for VCI in patients; 6 articles 


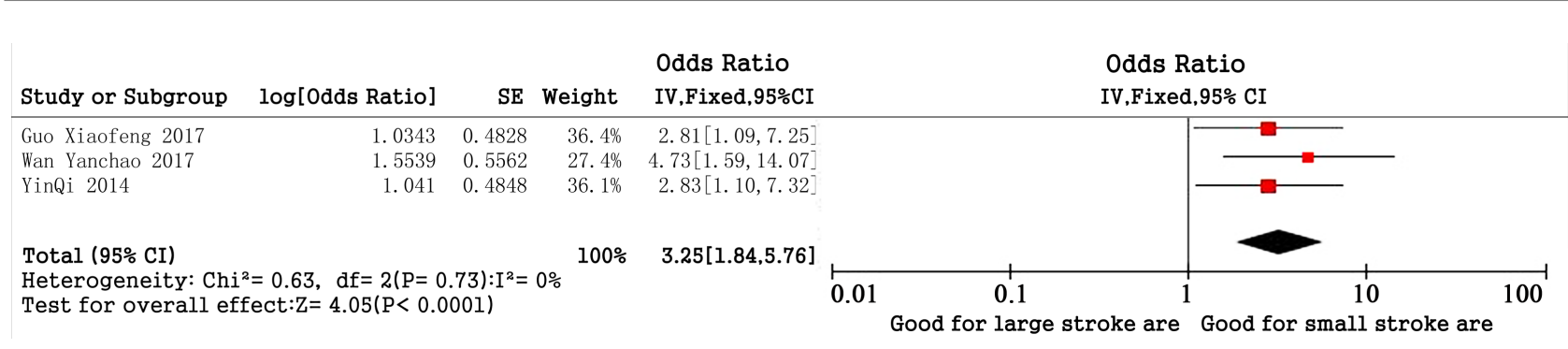

Figure 1. Relationship between stroke area and cognitive dysfunction.

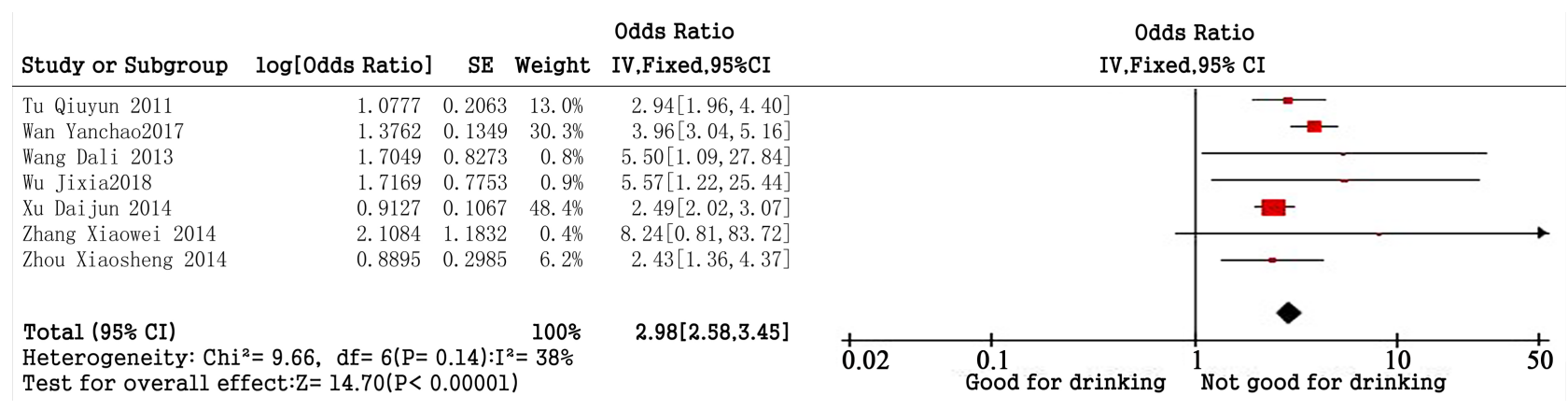

Figure 2. Relationship between drinking and cognitive dysfunction.

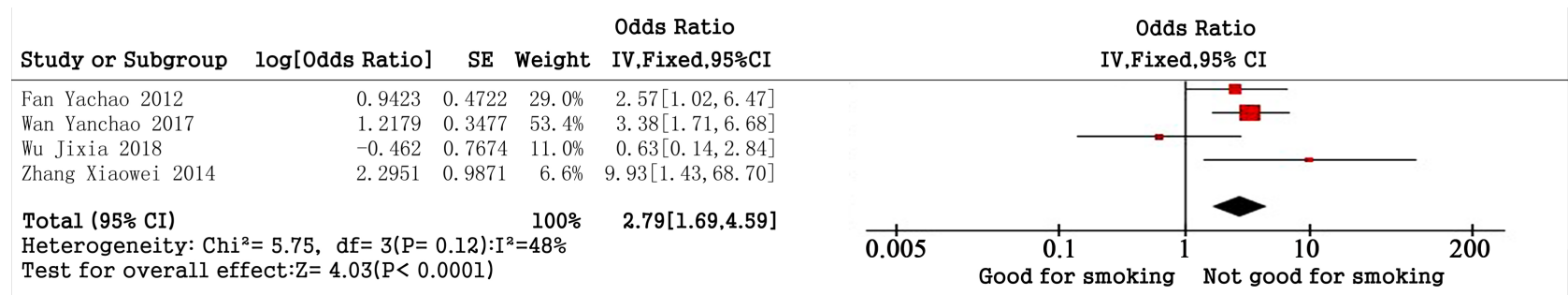

Figure 3. Relationship between smoking and cognitive dysfunction.

reported the connection of high homocysteine levels and VCI, 3 are available for meta-analysis. The merged results show that hyperhomocysteinemia is highly correlated with VCI and is a risk factor. $(\mathrm{OR}=2.35,95 \% \mathrm{CI}(1.93,2.85), P<$ $0.00001, \mathrm{I}^{2}=0 \%$ ) (Figure 4); 17 articles having reported that hypertension and hypertension history are related to VCI in patients, 8 cases are available for meta-analysis through heterogeneity and sensitivity, with the merged results showing that the history of hypertension is a risk factor for $\mathrm{VCI}(\mathrm{OR}=2.25,95 \% \mathrm{CI}$ $(1.86,2.71), P<0.00001, \mathrm{I}^{2}=0 \%$ ) (Figure 5); 15 article having reported that diabetes is associated with VCI, among which, 15 can be analyzed with meta-analysis. The merged results are $\mathrm{OR}=2.14,95 \% \mathrm{CI}(2.10,2.18), P<0.00001$, $\mathrm{I}^{2}=15 \%$ (Figure 6), indicating that diabetes is a risk factor for VCI; 15 articles that reported the correlation of VCI and the age of patients with cerebral infarction, only 5 are combined with effectors for meta-analysis. The merged results show that the advanced age is a risk factor for VCI. (OR $=1.82,95 \%$ CI $(1.62$, 2.03), $P<0.00001, \mathrm{I}^{2}=0 \%$ ) (Figure 7); 8 reports having reported that the history of cerebral infarction is associated with VCI in patients with cerebral infarction, 5 are available for meta-analysis. The merged results are OR $=1.54,95 \%$ CI. 
Odds Ratio

Study or Subgroup log[Odds Ratio] SE Weight IV,Fixed,95\%CI

\begin{tabular}{lllll}
\hline Feng Qinchun 2017 & 0.7812 & 0.133 & $55.5 \%$ & $2.18[1.68,2.83]$
\end{tabular}

Ning Fangbo $2011 \quad 0.9884 \quad 0.1667 \quad 35.3 \% \quad 2.69[1.94,3.73]$

Yin Songli 2014

$0.7678 \quad 0.3267 \quad 9.2 \%$

2. $16[1.14,4.09]$

Total (95\% CI)

Heterogeneity: $\mathrm{Chi}^{2}=1.02, \mathrm{df}=2(\mathrm{P}=0.60): \mathrm{I}^{2}=0 \%$

Test for overall effect: $Z=8.61(P<0.00001)$
$2.35[1.93,2.85]$

IV,Fixed,95\% CI

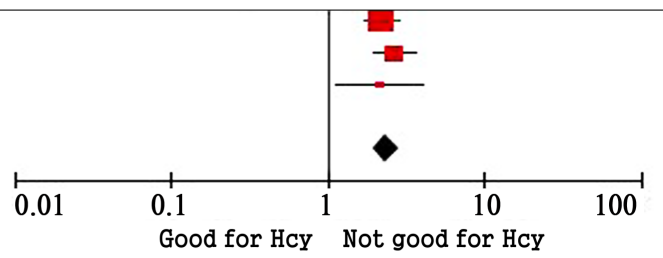

Figure 4. Relationship between HCY and cognitive impairment.

Odds Ratio

Study or Subgroup log[Odds Ratio] SE Weight IV,Fixed,95\%CI

\begin{tabular}{lllll}
\hline Fan Yachao 2012 & 0.8007 & 0.408 & $5.4 \%$ & $2.23[1.00,4.95]$
\end{tabular}

Gao Yuan 2015

Liu Yanjun 2010

Ning Fangbo 2011

Sun Yao 2018

Wang Dali 2013

Wang Jin 2017

Yin Songli 2014 $\begin{array}{lll}0.9513 & 0.3203 & 8.8 \%\end{array}$

$\begin{array}{lll}0.9869 & 0.4453 & 4.6 \%\end{array}$

$0.7585 \quad 0.1247 \quad 58.2 \%$

$\begin{array}{lll}0.9757 & 0.4862 & 3.8 \%\end{array}$

$\begin{array}{lll}1.1684 & 0.5019 \quad 3.6 \%\end{array}$

$\begin{array}{lll}0.6901 & 0.3496 & 7.4 \%\end{array}$

$0.7953 \quad 0.3327 \quad 8.2 \%$

$2.59[1.38,4.85]$

2. $68[1.12,6.42]$

2. $14[1.67,2.73]$

2. $65[1.02,6.88$

3. $22[1.20,8.60]$

1. $99[1.00,3.96]$

2. $22[1.15,4.25]$

Total (95\% CI)

Heterogeneity: $\mathrm{Chi}^{2}=1.27, \mathrm{df}=7(\mathrm{P}=0.99): \mathrm{I}^{2}=0 \%$

Test for overall effect: $Z=8.51(P<0.00001)$

$2.25[1.86,2.71]$

Odds Ratio

IV,Fixed,95\% CI

Figure 5. Relationship between hypertension and cognitive dysfunction.

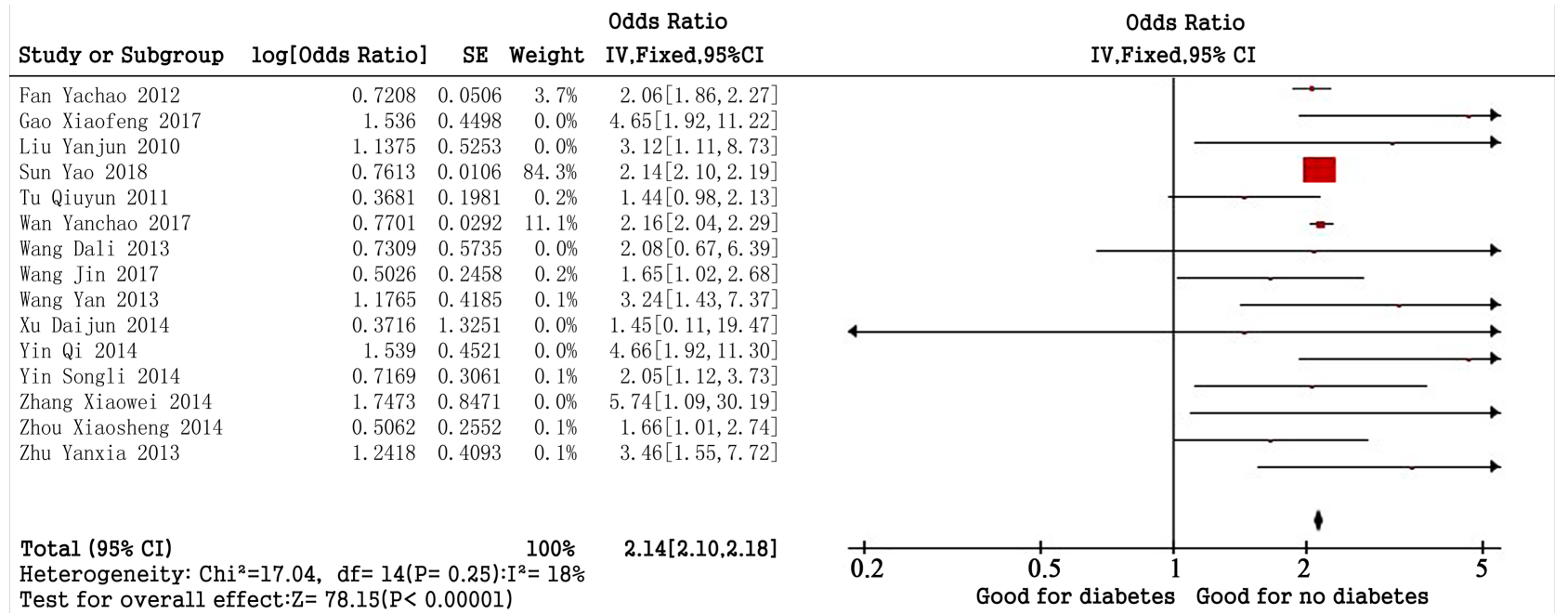

Figure 6. Relationship between diabetes and cognitive dysfunction.

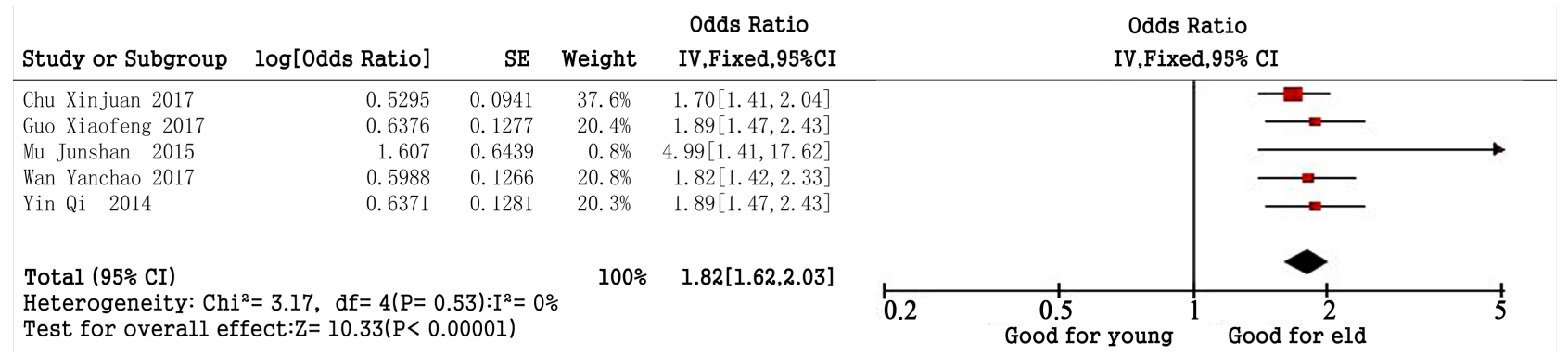

Figure 7. Relationship between age and cognitive dysfunction. 
(1.24, 1.92), $P=0.0007, \mathrm{I}^{2}=37 \%$ (Figure 8), which show that the history of cerebral infarction is a risk factor for VCI in patients; 8 articles having reported that hyperlipemia is associated with VCI in patients with cerebral infarction, among which, 3 articles are available for meta-analysis. The merged results show that hyperlipidemia is highly correlated with VCI as one of the risk factors. (OR $=1.45,95 \%$ CI $\left.(1.34,1.56), P<0.00001, \mathrm{I}^{2}=37 \%\right)$ (Figure 9). Of 17 reports about the connection of the education level and VCI in patients with cerebral infarction, 8 could be extracted for meta-analysis. The merged results show that $\mathrm{OR}<1$, so the degree of education is negatively correlated with VCI, which means the higher the degree of education is, the lower the probability of VCI appears. $\left(\mathrm{OR}=0.83,95 \%\right.$ CI $\left(0.78,0.89, P<0.00001, \mathrm{I}^{2}=36 \%\right)$ (Figure 10) Therefore, low education is a risk factor for VCI. Of 6 articles having reported the correlation of gender and VCI, 5 could be integrated for meta-analysis. The merged results show that $\mathrm{OR}=0.81,95 \% \mathrm{CI}(0.60,1.09), P=0.16, \mathrm{I}^{2}=0 \%, P>$ 0.05 , which is statistically insignificant, as a result, gender is not a risk factor for VCI (Figure 11).

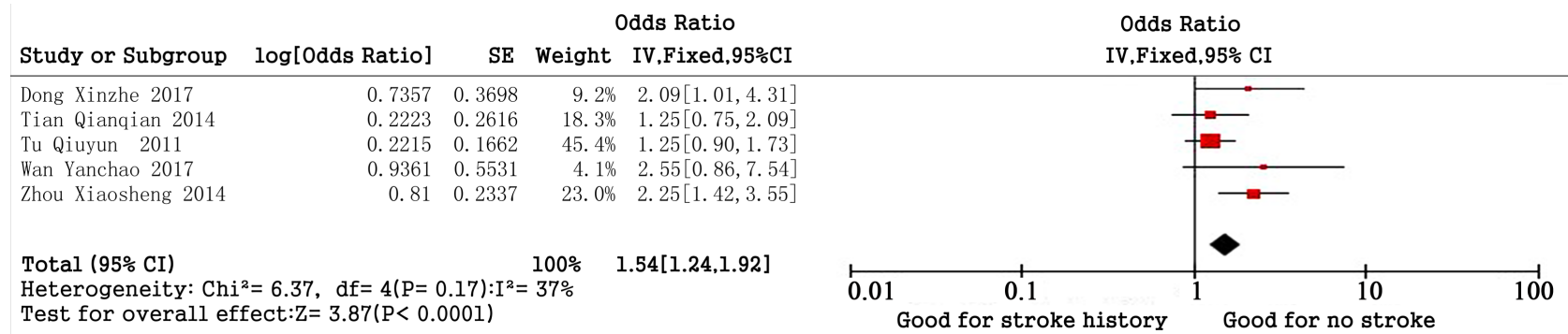

Figure 8. Relationship between stroke history and cognitive dysfunction.

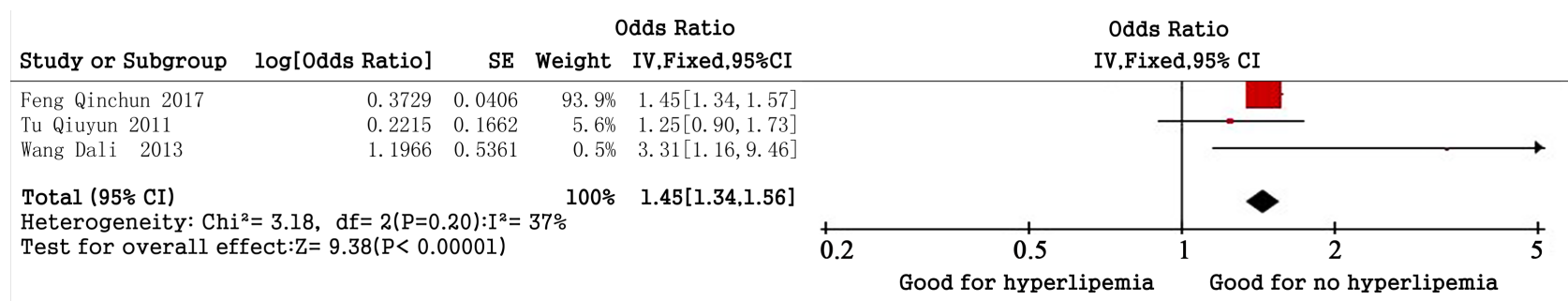

Figure 9. Relationship between hyperglycemia and cognitive dysfunction.

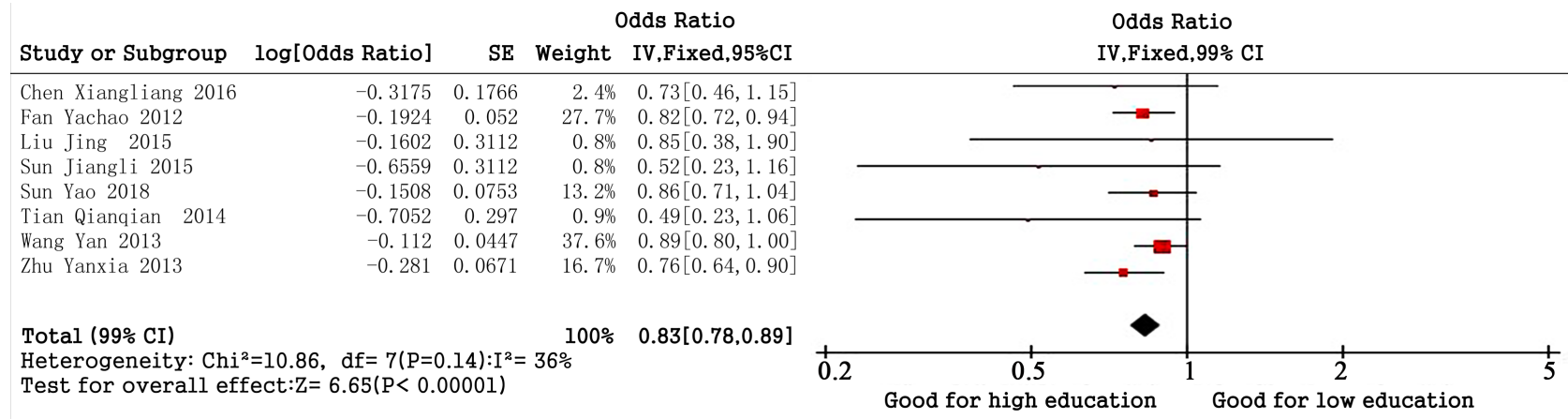

Figure 10. Relationship between education and cognitive dysfunction. 


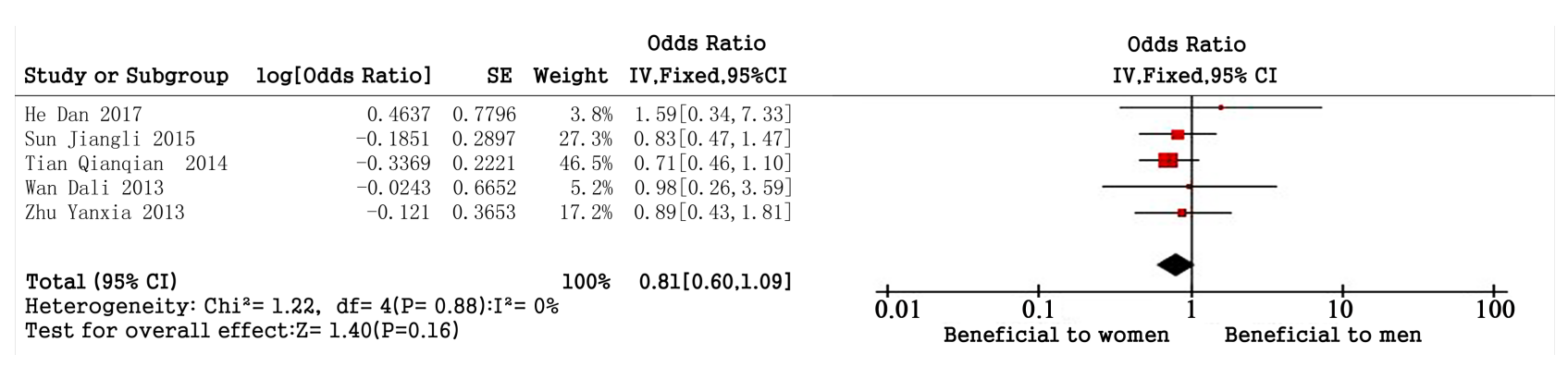

Figure 11. Relationship between gender and cognitive impairment.

\section{Discussion}

At present, with the increase of the aging population in China, there is a tendency of increased incidence rate of acute cerebral infarction. In the early stage of clinical nursing work, the nurse should assess the disease, identify the risk factors and take reasonable and effective intervention measures in time, which is of significance in clinic to help patients to recover cognitive function. Through the meta-analysis of the literatures, the factors that cause VCI in patients can be divided into two categories: vascular factors and patient factors, of which infarct size, drinking, smoking, hyperhomocysteinemia, diabetes, hypertension, Hyperlipidemia, history of stroke, and advanced age are the major risk factors for VCI. While, high education is a protective factor for VCI in patients. It is recommended that the above risk factors should be included in the patient's routine assessment.

According to the statistical principle, the greater the $\mathrm{OR}$ value is, the greater the correlation degree is. In this study, the infarct size is considered as the primary risk factor for VCI by analyzing the OR value of the combined benefit. The foreign scholars believe that large areas of lesions involved in the cerebral cortex and subcortical tissue will cause a complete decline in brain function, so that cognitive dysfunction may appear [32]. In particular, recurrent cerebral infarction can affect the cognitive function of patients more likely, because of the interaction between nerve damage and vascular injury and the cortical hypoperfusion of brain tissue, which make the neurological function abnormal [33]. In the studies of Sun Jiangli and other experts, $33.88 \%$ of patients with VCI have a history of cerebral infarction, which is significantly higher than the cognitive normal group [16]. Therefore, the history of cerebral infarction is also one of the risk factors for VCI, which suggests that once we have an acute cerebral infarction, we must actively prevent the enlargement of the area at first. We should assist the patient to lie on the back without the pillow, move the head toward the rear of the car, comfort the patient to relieve the mental vasospasm, and take the treatment of expansion tubes and other preventive measures to ensure blood supply and oxygen providing for the brain tissue. Give oxygen actively for the patients with a history of cerebral infarction, increase blood perfusion of brain tissue, and reduce nerve and blood vessel damage and the incidence of VCI.

The results of the meta-analysis of this study show that long-term high intake 
of alcohol is another risk factor for VCI [34]. It has been reported in the literatures that the risk of suffering from VCI for the patients of drinking alcohol is five times that of those who do not drink [26]. Moreover, there is a study for the patients in Chongqing on the incidence and risk factors of vascular cognitive impairment after three months of ischemic stroke, which shows that daily drinking can increase the risk of VCI after 3 months of the onset of cerebral infarction. Although the current mechanism of ethanol-induced cognitive dysfunction is not very positive, it is reported according to scholars that ethanol is a fat-soluble substance and there is a high affinity its brain tissue. If drinking alcohol for a long time, ethanol will continue to act on the brain, which has toxic effects on central nervous cells, causing changes in the number and conformational configuration of neurotransmitters in the synaptic cleft of the nervous system. It is the immediate cause of damaging the cognitive function [35]. Of course, the more alcohol intake, the stronger the toxic affects will be. Therefore, patients with long-term of great alcohol consumption, once acute cerebral infarction occurs, the blood supply of brain tissues will slow down as well as the cerebral cortex ischemia and hypoxia, which will quickly induce and aggravate cognitive impairment. In the clinical medical treatment and nursing care in the future, more special attention should be paid to patients with acute cerebral infarction who have a long-term of great alcohol consumption history. It is also recommended that scholars should pay more attention to the research on the amount of alcohol and time thresholds that can cause VCI so as to guide the health education in the future.

The results of this study show that elevated homocysteine is the 4th risk factor for VCI. Scholars believe that since homocysteine is a sulfur-containing amino acid, it can directly damage nerve cells related to cognitive function, leading to methylation and redox disorders in nerve cells. It can also activate and open the channel of NMDA receptor ions, promoting the large amount of calcium ions entering into the cell, facilitating the obvious accumulation of amyloid precursor protein, phosphorylated $\mathrm{AB}$ amyloid protein and tau protein, accelerating the production of a large amount of reactive oxygen species by COS-2, and stimulating the occurrence of the neurodegeneration and apoptosis, which lead to the occurrence of VCI [36]. In view of the above reasons, it should know the content of homocysteine and give the treatment timely when facing the patients with acute cerebral infarction

The meta-analysis of this study shows that patients with acute cerebral infarction who have a history of smoking, diabetes, hypertension, and hyperlipidemia have a higher risk of suffer from VCI, which is consistent with that of foreign studies. It is reported in Lu, D and Mizrahi, EH that metabolic syndrome is an important risk factor for VCI after acute cerebral infarction [37] [38]. For example, diabetes can quadruple the risk of cognitive decline, which can aggravate brain atrophy and cognitive dysfunction [39]. The data show that the incidence of VCI in diabetic patients is 2 to 4 times higher than that in non-diabetic patients after cerebral infarction. The reason of these risk factors leading to the 
main mechanism of VCI is that the blood lipid metabolism is abnormal and the blood vessel wall pressure has been increased, which result in the abnormality of the structure and function of the vessel wall, so that the aortic wall will thicken, and atherosclerosis as well as the glassy degeneration of microvessels occur [40]. It can also cause the change of hemodynamics and hemorheology, resulting in the decrease of the brain tissue perfusion and transportation barriers of nutrients such as oxygen and glucose, which will ultimately cause neuronal degeneration, necrosis and cerebrovascular events, increasing the risk of VCI. Therefore, special precautions should be taken by such patients.

The results also show that the risk of VCI in patients with cerebral infarction will increase with the growth of age and it is negatively correlated with the level of education, which means, the lower the educational level is, the higher the prevalence rate is [41]. Because there is negatively correlated between the increase of patients' age and their immunity and resistance, so that the physiological functions are inevitably declining, especially the brain mass and volume. Brain cell decline is the main cause of cognitive dysfunction. Therefore, the older the patient is, the higher the risk of VCI develops [42]. On the other hand, aging itself is usually accompanied by memory impairment, and the combined effects of aging and inflammation can significantly increase the decline in cognitive function after stroke [43]. At the same time, cognitive ability relies on complex cerebral cortical function and is significantly influenced by education. The lower the education level is, the less comprehensive understanding of cerebral infarction complicated with VCI appears, lacking of prevention awareness and behavior, so as to increase the incidence of VCI. Although the patient's age and education level are risk factors that cannot be changed, we can still value the assessment of such patients, with early detection and intensive care and guidance.

It is worth noting that a large number of researches have reported that white matter lesions are associated with VCI in patients with cerebral infarction, but they are not included in this study because of different research methods and the poor quality of the literatures [19] [44] [45]. A single study included reports that the degree of white matter lesions was positively correlated with VCI in patients with cerebral infarction. The higher the grade of white matter lesions is, the more severe the VCI occurs. The level I of white matter lesions is not related to the VCI of patients with cerebral infarction, but Level II, Level III and level IV are related [7]. Therefore, the relationship between white matter lesions as well as leukoaraiosis and VCI in patients with cerebral infarction should be further studied in the future, with research and analysis in grades. In addition, the results of this study show that gender is not associated with VCI in patients with cerebral infarction, which is different from previous studies. In the previous studies, a case-control study found that gender is associated with VCI by logistic regression analysis, but it did not clearly indicate that gender is a risk factor for cognitive dysfunction [30], without data available. Another case-control study indicated that males account for $57.38 \%$ of patients with cognitive dysfunction [16] and only $46.05 \%$ of those with normal cognition, which show that males are 
closely related to cognitive dysfunction. There is also a cross-sectional study indicating that there is a higher risk of suffering from VCI for males than females [13]. On the contrary, a case-control study clearly indicates that females are at risk of VCI after cerebral infarction [46] (OR $=6.477,95 \%$ CI $(1.275,32.902)), \mathrm{P}$ $=0.024)$. In the report of $\mathrm{Tu}$, Q. et al., it is also pointed out that females are more likely to suffer from VCI than male, but there is no significant difference. It can be seen that the results of previous studies are not consistent [41], and the results of this meta-analysis clearly define that gender is not a risk factor for VCI in patients with acute cerebral infarction.

\section{Summary}

In summary, there are 10 major risk factors for VCI in patients with acute cerebral infarction. All patients with acute cerebral infarction must actively clear the blockage of cerebral blood vessels at first, ensure the blood supply of the brain tissue and prevent the infarct size from expanding. For the patients with vascular risk factors such as diabetes, hypertension, hyperlipidemia and homocysteine, we must detect early and intervene and control in time in clinical nursing work, take actively preventing recurrence in patients with a history of cerebral infarction, and strengthen health education and popularize disease knowledge for the risk factors such as drinking and smoking; help patients establish a healthy lifestyle, and for the uncontrollable risk factors such as age and education level, medical staff should clarify their harmfulness, giving timely assessment and special attention to ensure the quality of patients' lives. Early identification and intervention of the above risk factors are important for better prevention and the delay of cognitive impairment. It is recommended that scholars should pay more attention to the research on how to control the risk factors in the future.

\section{Conflicts of Interest}

The authors declare no conflicts of interest regarding the publication of this paper.

\section{References}

[1] Bowler, J.V. (2007) Modern Concept of Vascular Cognitive Impairment. British Medical Bulletin, 83, 291-305. https://doi.org/10.1093/bmb/ldm021

[2] GBD 2015 Mortality and Causes of Death Collaborators (2016) Global, Regional, and National Life Expectancy, All-Cause Mortality, and Cause-Specific Mortality for 249 Causes of Death, 1980-2015: A Systematic Analysis for the Global Burden of Disease Study 2015. Lancet, 388, 1459-1544.

[3] Yang, G.H., Wang, Y., Zeng, Y.X., et al. (2013) Rapid Health Transition in China, 1990-2010: Findings from the Global Burden of Disease Study 2010. The Lancet, 6, 1988-2015. https://doi.org/10.1016/S0140-6736(13)61097-1

[4] Sturm, J.W. (2004) Quality of Life after Stroke: The North East Melbourne Stroke Incidence Study (NEMESIS). Stroke, 35, 2340-2345.

https://doi.org/10.1161/01.STR.0000141977.18520.3b 
[5] Dong, X.Z. (2017) The Correlation between Cognitive Impairment and Uric Acid in Transient Ischemic Attack/Light Stroke. University of Qingdao, Qingdao.

[6] Feng, Q.C., et al. (2017) Logistic Regression Analysis of Factors Influencing Cognitive Function in Elderly Patients with Acute Ischemic Stroke. Chinese Journal of Disease Control, 21, 822-826.

[7] Zhang, X.W. (2014) Risk Factors of Vascular Cognitive Dysfunction in Patients with Acute Lacunar Infarction. Chinese Medical Sciences University, Shenyang.

[8] Wang, Y. (2013) A Cross-Sectional Study of Cognitive Dysfunction and Related Factors in Patients with Acute Ischemic Stroke. Chinese Journal of Modern Neuropathy, 13, 279-284.

[9] Wang, J. (2017) Screening of Vascular Risk Factors for Cognitive Dysfunction in High-Risk Patients with Cerebral Apoplexy. Chinese Journal of Modern Neuropathy, 17, 138-142.

[10] Zhou, X.S. (2014) Analysis of Related Factors of Vascular Cognitive Dysfunction in Early Stage of Cerebral Apoplexy. Chinese Journal of practical Neuropathy, 17, 6-9.

[11] Tu, Q.Y. (2011) Epidemiological Investigation of Vascular Cognitive Impairment after Ischemic Stroke. Chinese Journal of Gerontology, 31, 3576-3579.

[12] Liu, J. (2015) Study on the Influencing Factors of Cognitive Dysfunction in Patients with Ischemic Cerebral Apoplexy. Chinese General Practice Medicine, 18, 1361-1365.

[13] Tian, Q.Q. (2014) Screening of Non-Dementia Vascular Cognitive Dysfunction in High-Risk Population with Stroke in Community and Analysis of Related Factors. Chinese Journal of Cardiovascular and Cerebrovascular Diseases, 16, 452-455.

[14] Yin, Q. (2014) Risk Factors of Mild Vascular Cognitive Impairment in Elderly Patients with Ischemic Stroke. Chinese Journal of Gerontology, 34, 4466-4467.

[15] Guo, X.F. (2017) Influential Factors of Mild Vascular Cognitive Impairment in Elderly Patients with Ischemic Stroke. Chinese and Foreign Medical Research, 15, 45-46.

[16] Sun, J.L. (2015) Analysis of Risk Factors of Non-Dementia Vascular Cognitive Dysfunction in High-Risk Patients with Cerebral Apoplexy. Chinese Journal of Practical Neuropathy, 18, 5-7.

[17] Yin, S.L. (2014) Risk Factors of Vascular Cognitive Dysfunction in Early Post-Stroke Patients. Journal of Mudanjiang Medical College, 35, 41-43.

[18] Chen, X.L. (2016) Assessment and Risk Factors of Cognitive Impairment in Patients with Cerebral Apoplexy. Nanjing University, Nanjing.

[19] He, D. (2017) Clinical Characteristics and Influencing Factors of Non-Dementiavascular Cognitive Dysfunction after Lacunar Infarction. Chinese General Practice Medicine, 20, 1037-1041.

[20] Fan, Y.C. (2012) Risk Factors and Clinical Characteristics of Mild Cognitive Impairment in Patients with Ischemic Cerebrovascular Disease: Retrospective Case Series Study. International Journal of Cerebrovascular Diseases, 20, 564-569.

[21] Ning, F.B. (2015) Analysis of Related Factors in Patients with Vascular Cognitive Dysfunction after Ischemic Stroke. Chinese Journal of Clinicians, 9, 932-936.

[22] Xu, D.J. (2014) Analysis of Related Factors of Vascular Cognitive Impairment after Ischemic Stroke. Grass-Roots medicine in China, 21, 896-898.

[23] Chu, X.J. (2017) The Present Situation and Influencing Factors of Vascula Cognitive Dysfunction in Patients with Ischemic Stroke. Chinese Journal of Clinical Physicians, 45, 43-44. 
[24] Mu, J.S. (2015) The Present Situation and Influencing Factors of Vascular Cognitive Dysfunction in Patients with Ischemic Stroke. Practical Geriatrics, 29, 151-153.

[25] Wang, Y.C. (2017) Risk Factors of Vascular Cognitive Impairment in Ischemic Cerebral Apoplexy. Chinese Journal of Practical Neuropathy, 20, 30-33.

[26] Wu, J.X. (2018) Relationship between Ischemic Vascular Cognitive Impairment and Plasma Biochemical Predictors. Chinese Journal of Practical Neuropathy, 21, 188-192.

[27] Liu, Y.J. (2012) Detection Rate and Risk Factors of Cognitive Impairment after Ischemic Stroke. Lanzhou University, Lanzhou.

[28] Zhu, Y.X. (2013) Study on the Risk Factors and Biomarkers of Cognitive Impairment in Patients with Ischemic Stroke. Medical University of Tianjin, Tianjin.

[29] Gao, Y. (2015) Risk Factors of Mild Cognitive Impairment in Patients with Arteriolarocclusive Stroke. Chinese Journal of Cardiovascular and Cerebrovascular Diseases, 17, 393-395.

[30] Wang, D.L. (2013) Relationship between Serum Cystatin c and Vascular Cognitiveimpairment after Cerebral Infarction. Chinese Journal of Cardiovascular and Cerebrovascular Diseases, 15, 684-686.

[31] Sun, Y. (2018) Chinese Journal of Cardiovascular and Cerebrovascular Diseases. Psychologist, 24, 73-74.

[32] Pendlebury, S.T., Mariz, J., Bull, L., et al. (2013) Impact of Different Operations Definitions on Mild Cognitive Impairment Rale and MMSE and MOCA Performance in Transient Is Chemic Attack and Stroke. Cerebrovascular Diseases, 36, 355-362. https://doi.org/10.1159/000355496

[33] Chen, Q.Y., Zhu, M.H., Xu, X.Q., et al. (2014) Effect of Early Scalp Acupuncture Combined with Rehabilitation Training on Cognitive Impairment in Patients with Cerebral Apoplexy. Chinese Traditional Chinese Medicine Science and Technology, 21, 97-98.

[34] Shen, N.N., Chen, W., Zhao, R.L., et al. (2010) Mild Cognitive Impairment and Non-Dementia Vascular Cognitive Dysfunction. Journal of International Cerebrovascular Disease, 18, 691-695.

[35] Zhou, D.H., Wang, J.Y., Li, J., et al. (2005) Frequency and Risk Factors of Vascular Cognitive Impairment Three Months after Ischemic Stroke in China: The Chongqing Stroke Study. Neuroepidemiology, 24, 87-95. https://doi.org/10.1159/000081055

[36] Yu, Y.X. (2015) Chinese Molecular Neurology. Anhui Science and Technology Publishing House, Hefei, 420.

[37] Lu, D., et al. (2016) Vascular Risk Factors Aggravate Cognitive Impairment in First-Ever Young Ischemic Stroke Patients. European Journal of Neurology, 23, 940-947. https://doi.org/10.1111/ene.12967

[38] Mizrahi, E.H., et al. (2010) Diabetes Mellitus Predicts Cognitive Impairment in Patients with Ischemic Stroke. American Journal of Alzheimer's Disease \& Other Dementias, 25, 362-366. https://doi.org/10.1177/1533317510365343

[39] Ben Assayag, E., et al. (2017) Type 2 Diabetes Mellitus and Impaired Renal Function Are Associated with Brain Alterations and Post stroke Cognitive Decline. Stroke, 48, 2368-2374. https://doi.org/10.1161/STROKEAHA.117.017709

[40] Chen, X.R. and Nie, B.G. (2009) Effects of Atto Vartadine Calcium on Blood Lipid and Cognitive Function in Patients with Vascular Cognitive Impairment Complicated with Hyperlipidemia. Chinese General Practice Medicine, 12, 1382-1384.

[41] Tu, Q., et al. (2014) The Current Situation on Vascular Cognitive Impairment after 
Ischemic Stroke in Changsha. Archives of Gerontology and Geriatrics, 58, 236-247. https://doi.org/10.1016/j.archger.2013.09.006

[42] Li, X.Y. and Lu, J. (2010) Cognitive Dysfunction and Rehabilitation after Stroke. Chinese Journal of physical Medicine and Rehabilitation, 32, 956-957.

[43] Alexandrova, M.L. and Danovska, M.P. (2016) Cognitive Impairment One Year after Ischemic Stroke: Predictorsand Dynamics of Significant Determinants. Turkish Journal of Medical Sciences, 46, 1366-1373. https://doi.org/10.3906/sag-1403-29

[44] Xun, L. (2009) Chinese General Practice Medicine. Chinese General Practic Medicine, 26, 234-236.

[45] Tao, Z.H. (2014) Study on Risk Factors of Vascular Cognitive Dysfunction after Cerebral Infarction. Journal of practical Cardiocerebral Pulmonary Angiopathy, 22, 17-19.

[46] Chen, X.L. (2016) Chinese Journal of Geriatric Heart Brain and Vessel Diseases. Nanjin University, Nanjin. 